\title{
Developing a vaccine for Haemophilus influenzae serotype a: Proceedings of a workshop
}

\author{
AD Cox ${ }^{1 *}$, L Barreto1, M Ulanova², MG Bruce ${ }^{3}$, RSW Tsang ${ }^{4}$ on behalf of the Conference \\ contributors $^{5}$
}

\section{Abstract}

Since the late 1990s there has been an emergence of Haemophilus influenzae serotype a (Hia) infections, especially in Indigenous communities in the northern regions of Canada and Alaska associated with significant morbidity and approximately a $10 \%$ mortality. A Hia vaccine could potentially prevent this disease and save the health care system millions of dollars in both acute and long-term care.

On March 23-24, 2016, the National Research Council (NRC), the Public Health Agency of Canada (PHAC) and the Canadian Institutes of Health Research (CIHR) co-organized a meeting on $\mathrm{H}$. influenzae serotype a (Hia) to examine the current state of disease epidemiology and a potential vaccine solution path. The meeting included representatives from academia, federal and territorial public health units, hospital laboratories, federal departments involved in Aboriginal health, advocacy organizations for Indigenous peoples and industry.

Representatives from industry confirmed having the capacity and the interest to support preparation of clinical trial batches. Canadian regulatory authorities have expressed a willingness to help ensure appropriate measures are in place for licensure purposes.

Furthermore, there is the capacity and interest in performing some clinical trials in Indigenous communities in both Canada and Alaska. Recommendations for next steps included: complete pre-clinical studies, improve epidemiological surveillance to better understand the extent of the disease in the rest of North America and globally, establish engagement mechanisms with national Indigenous organizations to ensure their peoples are fully involved in the process and explore funding opportunities to prepare clinical lots and undertake clinical trials.

\section{Affiliations}

${ }^{1}$ Human Health Therapeutics Portfolio, National Research Council, Ottawa, ON

${ }^{2}$ Northern Ontario School of Medicine, Lakehead University, Thunder Bay, ON

${ }^{3}$ Artic Investigation Program, Division of Preparedness and Emerging Infections, US Centers for Disease Control and Prevention (CDC), Anchorage, AK

${ }^{4}$ National Microbiology Laboratory, Public Health Agency of Canada, Winnipeg, MB

${ }^{5}$ All conference contributors are noted at the end of the paper

*Correspondence: andrew.cox@ nrc-cnrc.gc.ca

Suggested citation: Cox AD, Barreto L, Ulanova M, Bruce MG, Tsang RSW on behalf of the Conference contributors. Developing a vaccine for Haemophilus influenzae serotype a: Proceedings of a workshop. Can Commun Dis Rep. 2017;43(5):89-95. https://doi.org/10.14745/ccdr.v43i05a02

\section{Introduction}

Haemophilus influenzae is a Gram-negative bacterium that can cause a range of infections from otitis media to sepsis. $H$. influenzae strains include those that have on their surfaces polysaccharide capsules which divide the strains antigenically into six different capsular serotypes (a to f) and those without capsules (termed non-typeable). In the past, $H$. influenzae serotype $b$ (Hib) was the most common cause of meningitis in children until a glycoconjugate vaccine based upon the Hib polysaccharide capsule became part of the routine infant immunization schedule in Canada and the United States (US) in the early 1990s (1). Although the Hib vaccine has been very effective in decreasing Hib infections, since the late 1990s there has been a growing number of cases of $\mathrm{H}$. influenzae serotype a (Hia), especially in Indigenous communities in the northern regions of Canada and Alaska (2). The infection with Hia can be severe. In a recent study among young children, $42 \%$ reported meningitis, $19 \%$ bacteremic pneumoniae, $25 \%$ bone, joint and soft tissue infection and $11 \%$ died (3). There is currently no vaccine.

On March 23-24, 2016, the National Research Council (NRC), the Public Health Agency of Canada (PHAC) and the Canadian Institutes of Health Research (CIHR) co-organized a meeting on Hia to examine the current state of disease epidemiology and potential vaccine solution path. It included representatives from academia, federal and territorial public health units, hospital laboratories, federal departments involved in Aboriginal health, advocacy organizations for Indigenous peoples and industry.

This article summarizes the information shared during this meeting by highlighting recent trends in the epidemiology of Hia in Canada and elsewhere, discusses how Indigenous peoples have and can be involved in Hia vaccine development, summarizes Hia immunology and pre-clinical Hia vaccine research, considers different options for vaccine development and identifies recommendations for moving forward. 


\section{Epidemiology of Haemophilus influenzae serotype a}

\section{Hia globally: An overview}

\author{
Dr. Raymond Tsang
}

To investigate the global presence of $\mathrm{Hia}$, an interrogation of the $H$. influenzae Multi-Locus Sequence Typing website (4) was performed on March 4, 2016. From this data set it was apparent that Hia has been observed in North and South America, Africa, Europe, Asia and Australia. The earliest documentation of Hia infection was in the Gambia, Papua New Guinea and the Dominican Republic in 1980, indicative of a global distribution of this pathogen. Similarly, the Multi-Locus Sequence Typing website also described a cluster of invasive Hia disease cases in New Mexico in 2009-2010 which was found to be caused by a strain of Hia with the same sequence type as a strain seen in Alaska (5).

There is some evidence that since the introduction of Hib vaccine, the incidence of Hia has been rising in certain regions. For example, in a population-based study in Utah in children under 18 years old from 1998 to 2008 (6), the incidence rate of Hia disease cases increased from $0.8 / 100,000$ to $2.6 / 100,000$. In a similar study, the incidence of invasive Hia disease in those over 65 years old was 2.7/100,000 (7). In Brazil, before the introduction of the Hib conjugate vaccine, $97.8 \%$ of all invasive $H$. influenzae disease was due to Hib, while Hia accounted for only $0.5 \%$ of the disease (8). Five years after the introduction of the Hib conjugate vaccine, the overall incidence of Hib meningitis in the general population had decreased from 2.39 to 0.06 per 100,000 . However, transient serotype replacement by two clones of Hia was observed (9). The changing epidemiology of invasive $H$. influenzae disease globally requires further study.

\section{Hia in Canada: A case series \\ Dr. David Scheifele}

The Immunization Monitoring Program ACTive (IMPACT) is an active, hospital-based surveillance network based at 12 children's hospitals across Canada. IMPACT hospitals account for over $90 \%$ of tertiary care pediatric beds in Canada $(10,11)$. Invasive $H$. influenzae disease (all serotypes) has been monitored since 2007. A case is defined by an isolate obtained from a normally sterile site and is confirmed as serotype Hia by the National Microbiology Laboratory (NML, PHAC, Winnipeg). The cases observed are a combination of provincial-origin cases and those referred from the territories.

A total of 102 Hia cases have been observed since 2007, an average of 12.5 cases per year, with territorial referrals representing one-third of the cases. Most cases were observed in Winnipeg, Edmonton and Montréal, which serve as referral centers for the territories. For the provincial-origin cases it was possible to determine from the postal-code information linked to each case that there was considerable geographic spread, but a key observation was that this disease was not just a far northern risk. Based upon the pediatric cases observed, it was clear that greater than half were in infants less than one year old, some just a few weeks old. Approximately two-thirds of the cases occurred before two years of age. The illness was often severe, requiring intensive care; nine percent died as a result of the infection. Almost all cases were of Indigenous origin. In terms of severity, half of those infected developed meningitis, while pneumonia, otitis media and septic arthritis were also prominent. Over half of those with meningitis experienced seizures, reflecting advanced infection at diagnosis.

\section{Hia in Quebec}

Dr. Andrée-Anne Boisvert

Invasive Hia infections are a significant health problem for Indigenous children living in Northern Quebec, specifically Nunavik and James Bay Cree where 40 of the 62 cases from 2006-2015 have been observed (2). The majority of cases are seen in the young with $66 \%$ being less than five years of age. This corresponds to a peak of approximately 100 cases per 100,000 people for all age groups, 225 per 100,000 in those less than five years of age and 350 per 100,000 in those less than one year. In the James Bay Cree territory, these numbers are higher than was observed for Hib disease in the pre-vaccine era. The mortality rate is $15 \%$ and all deaths were in the Inuit population. The cases almost routinely involve helicopter evacuation (Medi-Vac) to an urban centre.

\section{Hia in Nunavut}

\section{Dr. Kim Barker}

The population of Nunavut is 32,000 , of which $90 \%$ are Inuit. Nunavut has a publicly funded vaccine for Hib. The majority of all invasive $H$. influenzae cases reported from 2007 onwards have been due to Hia (over 60\%) and within the Hia cases over $90 \%$ were less than two years old. There was a slight preponderance of male cases becoming ill (3). Rates of Hia are generally similar among the regions of Nunavut, except for certain years in which Kitikmeot showed a spike in incidence. These spikes correspond to rates of 140-160 cases per 100,000.

The severity of the disease progression is rapid. Due to the remoteness of some regions and the rapidity with which the disease progresses, there is a total reliance upon helicopter evacuation (MediVac) for every child that contracts invasive Hia disease. At an average of six cases per year, and a cost of $\$ 55 \mathrm{~K}$ for each evacuation, transportation costs alone for Hia in Nunavut is approximately $\$ 0.33 \mathrm{M}$ each year.

\section{Hia in Alaska}

Dr. Michael Bruce

Since 2000, there have been over 50 cases of invasive Hia disease in Alaska (5) (and MGB's unpublished data). This corresponds to $60 \%$ of all $\mathrm{H}$. influenzae disease occurring in Alaska. Of those cases, $85 \%$ were concentrated in the western region of the state. The disease is mostly observed in the very young with $33 \%$ being less than seven months old, $67 \%$ being less than one year old and $83 \%$ less than 2 years old. Eight months is the mean age of invasive Hia cases; $67 \%$ are male, with $92 \%$ of Indigenous background. These correspond to annual incidence rates of 13 per 100,000 for those less than two years old and 44 per 100,000 for Alaskan Natives less than two years 
of age. Over $90 \%$ of invasive Hia cases were age-appropriately vaccinated for $\mathrm{Hib}$ and over $80 \%$ of those taken ill required hospitalization with an eight percent case fatality rate. Due to the remote location, air transport was needed for $80 \%$ of cases. There has been little variation in the three sequence types (ST 576, ST 23 and ST 56) observed over the past decade, with ST56 dominating recently (5). The choice of carrier protein was also critical to the success of the Hib vaccine in Alaska as a switch to the outer membrane protein complex (OMPC) of Neisseria meningitidis from diphtheria toxoid as the carrier protein was necessary to generate protective titers (12) and a subsequent shift from OMPC to a genetic variant of diphtheria toxoid (CRM) as the carrier facilitated a breakthrough of Hib disease that was only corrected by a return to OMPC as the carrier protein $(13,14)$.

\section{Engaging Indigenous people in Hia vaccine development}

Early engagement with Indigenous peoples is absolutely vital in order to ensure that the communities who are currently at the greatest risk of this emerging pathogen are involved and aware of the threat and possible solution.

\section{ClHR and "two-eyed seeing" in First Nations, Inuit and Métis research}

Dr. Simon Brascoupé

The priorities of the CIHR include enhanced patient experiences and outcomes through health innovation, health and wellness for Aboriginal peoples, a healthier future through preventive action and improved quality of life for persons living with chronic conditions. These priorities are to be considered in parallel with the strategic direction of the Institute of Aboriginal Peoples' Health which is: First Peoples and communities driving First Nations, Inuit and Métis (FNIM) health research and knowledge translation, in order to transform First Peoples' health through Indigenous ways of knowing and wellness, strength and resilience for First Peoples.

It is clear that these priorities resonate strongly with the development of a Hia glycoconjugate vaccine. However, it is necessary to ensure that this development is done with the Indigenous populations and considering the Indigenous perception of health and wellness. This will require a move from research on FNIM to research with and by FNIM. It is vital to deploy "two-eyed seeing", i.e., a western viewpoint and an Indigenous perspective, to create a new norm of excellence in FNIM research, enhancing relevance of community research priorities and FNIM knowledges, values and cultures.

\section{Assembly of First Nations engagement principles}

Dr. Marlene Larocque

The Assembly of First Nations (AFN) is a policy body which supports First Nations' Health. It will generate information with partners into better outcomes for First Nations. The engagement principles for partnerships with AFN include the following: policies/programs with First Nations involvement work better, the earlier the engagement, the better, the $\mathrm{CIHR}$ guidelines on
Aboriginal health are a good resource, there is a need to have a holistic approach to protect Indigenous knowledge and consider Indigenous practices to keep children safe. It is also critical to recognize that the burden of disease may be influenced by the physical environment such as overcrowding and lack of access to safe, running water.

\section{Health Canada First Nations and Inuit Health Branch}

Kathleen Lydon-Hassan

Health Canada First Nations and Inuit Health Branch (FNIHB) works with its partners to improve health outcomes, provide access to quality health services and support greater control of the health system by First Nations and Inuit. The overall goal is for the federal, provincial, territorial governments and the First Nations and Inuit peoples to work together on a shared path to improved health. FNIHB supports immunization programming to on-reserve peoples by either directly delivering or financially supporting First Nations on-reserve communities and/or organizations in the delivery of immunization.

\section{Hia immunity and pre-clinical Hia vaccine research}

\author{
Naturally acquired immunity to Hia \\ Dr. Marina Ulanova
}

Researchers at the Northern Ontario School of Medicine have been working diligently to establish relationships with the First Nations population in the community and work closely with the Sioux Lookout Meno Ya Win Health Centre to examine the specificity of naturally acquired immunity to Hia. Northern Ontario is a region where invasive disease has a high incidence. Between 2002-2016, incidence rates of Hia infections in children less than five years old ranged from 7.7 to 23.2 per $100,000(15,16)$.

Intriguingly, the activity of naturally acquired bactericidal antibodies against $\mathrm{Hia}$ is higher in Indigenous compared to non-Indigenous adults, with geometric mean titers being 351 compared to 183 respectively and immunoglobulin M (lgM) being more prevalent than immunoglobulin $\mathrm{G}(\mathrm{lgG})$ in the Hia capsular polysaccharide specific antibody repertoire (17). Significantly however, this study clearly revealed that both Indigenous and non-Indigenous healthy adults have high titers of bactericidal antibodies to Hia, illustrating that Hia is present in the whole community and not just in the Indigenous members of the community (17).

Critically, it was clearly demonstrated that naturally acquired serum bactericidal activity against Hia is nearly $100 \%$ due to antibodies to the capsular polysaccharide. Bactericidal activity was readily removed via absorbance with purified capsular polysaccharide of Hia. Absorbance of sera with an antigenically cross-reactive polysaccharide of Streptococcus pneumoniae serotype 6B had no effect on the bactericidal titers against Hia, emphasizing the necessary specificity to achieve protection and 
providing clear support for the glycoconjugate vaccine based upon the capsular polysaccharide of Hia approach.

\section{Hia vaccine development research}

Dr. Andrew Cox

Pre-clinical research and development studies have been carried out in the NRC laboratories. Significant achievements have been made including the following:

- The growth of several Hia strains with non-bovine approved media in 30L fermenters and two PHAC established seed lot cell bank strains.

- A 400 mg/L yield of Hia capsular polysaccharide.

- Purity of capsular polysaccharide isolation has been confirmed by nuclear magnetic resonance spectroscopy.

- $\quad$ Sizing of capsular polysaccharide in readiness for conjugation has been optimized via sonication methodologies.

- Oxidation of the sized capsular polysaccharide has been achieved and degree of oxidation established by nuclear magnetic resonance spectroscopy.

- Conjugation has been optimized via direct reductive amination with several carrier proteins including human serum albumin, CRM and protein D.

- Immunogenicity of conjugates has been established by enzyme-linked immunosorbent assay (ELISA) following mice and rabbit immunizations.

- Functional activity of derived antisera has been illustrated with best titers observed with CRM as carrier protein in serum bactericidal assays, a recognized correlate of protection for Hib disease.

In addition to these achievements, no cross reactivity in terms of recognition or functionality was observed between Hia sera and Hib strains and vice-versa, illustrating specificity of the response and the need for a Hia specific vaccine. Proof of concept has been established as all Hia strains examined were killed by conjugate vaccine derived sera.

Further studies will focus on establishment of functional sera with acceptable adjuvants (e.g. alum) and the examination of levels of immunogenicity provoked by alternate carrier proteins.

\section{Considerations in vaccine development}

There are many key considerations to contemplate when moving a vaccine from the pre-clinical stage to the next stages of the development pathway. These include the ability to produce clinical material of good manufacturing practice (GMP) grade, ensuring that all regulatory considerations (be it quality assurance or clinical trial sizes) are in place and to have the necessary infrastructure to run the clinical trials.

\section{Clinical trial capabilities in Canada}

\section{Dr. Scott Halperin}

The Canadian vaccine research environment is supported and monitored by IMPACT $(10,11)$, the Canadian Association for Immunization Research and Evaluation (CAIRE) (18) and the Canadian Immunization Research Network (19). IMPACT's role is to establish the burden of a given disease, test vaccine effectiveness and safeguard vaccine safety monitoring. CAIRE's role is to encourage and enhance applied vaccinology research so that Canadians have timely access to new and improved vaccines. The Canadian Immunization Research Network is a multi-disciplinary network with a hundred researchers across Canada at more than 35 sites, supported by PHAC and CIHR. It has a focus on the late stage of the vaccine lifecycle from safety to programming. It provides a formal infrastructure for research and collaboration and its prime objectives are to test vaccine safety and effectiveness and maintain a rapid response capacity.

The Canadian Immunization Research Network operates a clinical trials network via a core network of clinical trial sites across Canada. It has the ability to conduct rapid trials in large and specialized groups, including pediatric and at-risk populations' capabilities and has done trials with the First Nations and Métis population (20).

The Canadian Immunization Research Network could provide the following contributions to Hia vaccine development: Burden of disease studies, Phase 1/Phase 2 clinical trials, modelling and cost effectiveness studies (MODERN), knowledge, attitudes, beliefs and behaviours studies and program evaluation.

\section{Regulatory considerations}

Dr. Richard Siggers

Beginning with the pre-clinical research and development stage and continuing through the post-market authorization period, awareness of regulatory considerations will facilitate product development efficiencies and bolster confidence in the safety and quality of marketed products. It is recommended that direct consultation with national regulatory authorities be considered at each stage of product development. For example, a meaningful approach to product characterization is critical to the success of manufacturing biologics. Product characterization is meant to identify key quality attributes of products shown to have an appropriate safety profile and to be efficacious in clinical studies. These key quality attributes will be used throughout the post-licensure stage as measures of product consistency and ensures that commercially manufactured product is representative of product tested successfully in clinical studies. Investing resources in product characterization early in the development stage will ensure that product quality and safety is not negatively impacted as the manufacturing process evolves from pilot scale to commercial scale.

The manufacturing of biologics (i.e. vaccines) is complex due to the inherent variability and diversity of starting materials, the complexity of the biological systems utilized in the manufacturing processes, the possibility of adventitious agent contamination and the numerous manufacturing processes which all have the potential of impacting product quality. Consequently, there is a need to apply quality control throughout the various stages of the manufacturing process to ensure the risk is not deferred to the final lot release tests which may not always be able to detect all biologically relevant changes in product quality. In addition, monitoring the manufacturing process mitigates the risk batch failures and market supply shortages. Setting appropriate specifications is critical to ensure product characteristics are maintained throughout the product's lifecycle. It is also important to note that similar products developed independently may have different quality specifications, as long as these specifications are 
supported by the quality attributes of the clinical lots shown to be safe and efficacious. In conclusion, knowledge of regulatory considerations and discussions with national regulatory authorities throughout the product development stages will help to avoid unnecessary delays and potentially expensive setbacks for manufacturers.

\section{Production of glycoconjugate vaccines by industry \\ Dr. Richard Kensinger, Dr. Don Gerson and Pradip Ghate}

Industrial representatives from Inventprise, PnuVax and Sanofi detailed that their facilities all have current GMP manufacturing capacity at a scale at least consistent with the requirements needed for the Hia vaccine and significant experience in the production of glycoconjugate vaccines.

In addition to the GMP requirements, the speakers highlighted the following key considerations that are often overlooked but critical to glycoconjugate vaccine production requirements including:

- Defining critical quality attributes at the start, before transfer to manufacturing.

- Thorough characterization of the seed lot.

- Ongoing monitoring of impurities as free polysaccharide is not the product.

- Assessing process scalability through physicochemical characterization to demonstrate product comparability.

- Ensuring adequate amounts of glycoconjugate vaccine. Estimated needs are: Phase I, 3-5g PS; Phase II, $10 \mathrm{~g}$ PS; Phase III, 10-100g PS depending upon dose requirements.

Each industrial representative confirmed that they had the capacity and/or the interest in contributing to the clinical lot production of this glycoconjugate vaccine.

\section{Public-private partnerships in vaccine development}

Drs. Mark Kane and Jason Crawford

Vaccine development globally has transitioned from a pseudo "cottage industry" where countries' public health institutes or local companies produced vaccines to one where it is perceived that "only Big Pharma can make a new vaccine". Organizations such as the Global Alliance for Vaccine and Immunization have made some progress in challenging the dogma that the developing world only gets a vaccine if it is profitable in the industrialized world, but much more needs to be done in this regard. Hia vaccine could follow the precedent set by the MenAfriVac vaccine for a new development model. The challenge, however, is that the Gates Foundation or the Vaccine Alliance are probably not interested in supporting a vaccine for potentially small populations in rich countries (US and Canada). Thus, the solution will be to identify federal government funding opportunities. It is also possible that Hia disease could expand in much the same way that the Hib disease did.
It was apparent that generating an advanced market commitment is a good strategy for this kind of product. It is important to start with the end in mind and try to establish who the recipients would be. It is probable that this product will warrant orphan vaccine status which would have implications for clinical trial monitoring and likely increase the need for increased post-market scrutiny. The vaccine could be ready for clinical market within two years and the opportunity to build upon the Hib vaccine experience will be tremendously helpful. It is imperative that this Hia vaccine is presented to national Indigenous organizations in order to partner and develop strategies with them to confirm the need and illustrate the potential of this product. It would be very useful to develop a working group structure to formalize efforts. This would include the development of a business plan to show that there would be a "benefit" to having the product available. This does not necessarily translate to profitability for the manufacturer, but has to illustrate what the benefit would be to the "investor" and, of course, the recipients. A modelling study on the cost-effectiveness and impact of such a vaccine would be crucial to help illustrate the value. There is a clear need to better define the burden of the disease.

Thus the key points to consider in terms of epidemiology, economics and vaccine development are:

- Do we have enough epidemiological data on burden in Indigenous and Alaskan native peoples?

- Do we have enough data from the US, Canada and the rest of the world? How can we encourage collaboration to get more global data?

- Do we have preliminary cost-effectiveness data? Who will carry out this modelling analysis?

- Do we have a strategy for consideration of what carrier protein to use? Would we consider two glycoconjugate vaccines with different carrier proteins, one for Canada and one for Alaska?

\section{Recommendations and next steps}

The workshop concluded with an all-participant roundtable to discuss all the matters raised during the presentations and to consider the key components to focus upon in order to effectively move this potential vaccine solution further along the development pathway.

The following is a list of the recommendations identified during the workshop:

1. Complete pre-clinical studies:

- The choice of protein carrier should be carefully evaluated as the Alaskan experience points to the importance of the carrier in order to induce protective immunity in infants less than six months old.

- The ability of adjuvants approved for human use (e.g. alum) to elicit protective titers needs to be established.

- There is a need to establish and obtain regulatory approval for bactericidal assay with human sera to illustrate laboratory correlate of protection with clinical trial studies. 
2. Improve epidemiological surveillance to better understand the extent of the disease in the rest of North America and Globally:

- $\quad$ Work with partners nationally and internationally (e.g. Pan American Health Organization) to better define global epidemiology.

3. Establish engagement mechanisms with national Indigenous organizations:

- Explore mechanisms to appropriately engage with AFN, Inuit Tapiriit Kanatami and other advocacy groups to ensure that Indigenous groups are involved in the process to set direction and make decisions.

4. Explore funding opportunities to prepare clinical lots and undertake clinical trials:

- $\quad$ Prepare a business plan/policy paper to demonstrate value of a Hia vaccine.

- Demonstrate economic burden of disease and develop modelling simulations to illustrate cost-effectiveness of a Hia vaccine solution.

- Formalize a working group to co-ordinate activities.

\section{Conclusion}

Following the introduction of the Hib vaccine in the mid-1980s, there has been a remarkable decrease in the number of reported cases of Hib disease. Since the late 1990s there has been a concomitant increase in Hia infections in Indigenous communities in Alaska and the northern regions of Canada. Young infants and children from our North American arctic communities continue to suffer and die from an infection that appears to be preventable. All the elements are now present to develop the Hia vaccine.

\section{Conflict of interest}

ADC, MGB, and RSWT have no conflicts of interest to declare.

MU holds an Investigator Initiated Research Grant from Pfizer for an unrelated study. LB is a Senior Scientific Advisor with Inventprise/InventVac, Redmond, Washington/Vancouver, British Columbia and a Strategic Advisor to NEOMED-LABS, Montreal, Quebec; and he has worked with the NRC Vaccine and Immune Therapeutics Group (HHT) from 2011-2015 and as a consultant for NRC in relation to Hia vaccine development. He worked with Sanofi Pasteur from 1988 to 2010 on Hib and combination vaccine development.

\section{Contributors}

Kim Barker, Department of Health, Government of Nunavut, lqaluit, NU

Luis Barreto, National Research Council, Ottawa, ON

Andrée-Anne Boisvert, Montreal Children's Hospital, Montréal, QC

Simon Brascoupé, Carleton University, Ottawa, ON
Michael Bruce, US Centers for Disease Control and Prevention (CDC), Arctic Investigations Program, Anchorage, AK, US

Cindi Corbett, Public Health Agency of Canada, Winnipeg, MB

Andrew Cox, National Research Council, Ottawa, ON

Jason Crawford, Centre for Drug Research and Development, Vancouver, BC

Don Gerson, PnuVax, Montréal, QC

Pradip Ghate, Inventprise, Seattle, WA, US

Scott Halperin, Dalhousie University, Halifax, NS

Mark Kane, Inventprise, Seattle, WA, US

Richard Kensinger, Sanofi, Swiftwater, PA, US

Marlene Larocque, Assembly of First Nations, Ottawa, ON

Isabelle Letourneau, Canadian Institutes for Health Research, Montréal, QC

Kathleen Lydon-Hassen, Health Canada, Ottawa, ON

David Scheifele, University of British Columbia, Vancouver, BC

Richard Siggers, Health Canada, Ottawa, ON

Raymond Tsang, Public Health Agency of Canada, Winnipeg, MB

Marina Ulanova, Northern Ontario School of Medicine,

Thunder Bay, ON

\section{Acknowledgments}

Many thanks to all the workshop participants who offered their time and input.

\section{Funding}

This meeting was organized by the National Research Council, the Public Health Agency of Canada and the Canadian Institutes of Health Research and hosted by the National Research Council. $\mathrm{CIHR}$ funded the travel of some academic participants and the NRC provided refreshments at breaks and funded the travel of the facilitator of the conference.

\section{References}

1. Public Health Agency of Canada [Internet]. Canadian Immunization Guide, Part 4, Active Vaccines, Haemophilus Influenzae Type B Vaccine [Internet]. Ottawa: PHAC [updated March 2017; cited March 24, 2017]. Available from: https:// www.canada.ca/en/public-health/services/publications/ healthy-living/canadian-immunization-guide-part-4-activevaccines/page-5-haemophilus-influenzae-type-b-vaccine. html.

2. Boisvert AA, Moore D. Invasive disease due to Haemophilus influenzae type A in children in Canada's north: A priority for prevention. Can J Infect Dis Med Microbiol. 2015;26:291-292.

3. Tsang RS, Li YA, Mullen A, Baikie M, Whyte K, Shuel M, Tyrrel G, Rotondo JA, Desai S, Spika J. Laboratory characterization of invasive Haemophilus influenzae isolates from Nunavut, Canada, 2000-2012. Int J Circumpolar Health 2016;75:29798. 
4. PubMLST [Internet]. Haemophilus influenzae MLST Databases [cited 2017 Mar 24]. Available from: http:// pubmlst.org/hinfluenzae/.

5. Bruce MG, Zulz T, DeByle C, Singleton R, Hurlburt D, Bruden D, Rudolph K, Hennessy T, Klejka J, Wenger JD. Invasive disease caused by Haemophilus influenzae serotype $a$, an emerging pathogen in Alaska. Emerg Infect Dis. 2013;19:932-937.

6. Bender JM, Cox CM, Motttice S, She RC, Korgenski K, Daly JA. Invasive Haemophilus influenzae disease in Utah children: An 11-year population-based study in the era of conjugate vaccine. Clin Infect Dis. 2010;50:e41-e46.

7. Rubach MR, Bender JM, Mattice S, Hanson K, Weng HYC, Korgenski K, Daly JA, Pavia AT. Increasing incidence of invasive Haemophilus influenzae disease in adults, Utah, USA. Emerg Infect Dis. 2011;17:1645-1650.

8. Zanelle RC, Casagrande ST, Bokermann S, Almeida SCG, Brandileone MCC. Characterization of Haemophilus influenzae isolated from invasive disease in Brazil from 1990 to 1999. Microbial Drug Resistance. 2002;8: 67-72.

9. Ribeiro GS, Lima JBT, Reis JN, Gouveia EL, Cordeiro SM, Lobo TS, Pinheiro RM, Ribeiro CT, Neves AB, Salgado K, Silva HR, Reis MG, Ko Al. Haemophilus influenzae meningitidis 5 years after introduction of the Haemophilus influenzae type b conjugate vaccine in Brazil. Vaccine. 2007;25:4420-4428

10. Scheifele DW, Halperin SA, and members of the Health Canada/CPS Immunization Monitoring Program Active (IMPACT). A model of active surveillance of vaccine safety. Semin Pediatr Infect Dis. 2003;14:213-219.

11. Bettinger JA. Halperin SA, Vaudry W, Law BJ, Scheifele DW. The Canadian Immunization Program ACTive (IMPACT): Active surveillance for vaccine adverse events and vaccine preventable diseases. Can Commun Dis Rep. 2014;40(S3):41-4. Available from: http://www.phac-aspc.gc.ca/publicat/ ccdr-rmtc/14vol40/dr-rm40s-3/comment-d-eng.php.

12. Ward J, Brenneman G, Letson GW, Heyward WL, Alaska H. Influenzae Vaccine Study Group. Limited efficacy of a Haemophilus influenzae type b conjugate vaccine in Alaska native infants. New Eng J Med. 1990;323:1393-1401.
13. Singleton R, Hammitt L, Hennessy $T$, Bulkow L, DeByle C, Parkinson A, Cottle TE, Peters H, Butler JC. The Alaska Haemophilus influenzae Type b Experience: Lessons in controlling a vaccine-preventable disease. Pediatrics. 2006;118:421-429

14. Bulkow LR, Wainwright RB, Letson GW, Chang WJ, Ward JI. Comparative immunogenicity of four Haemophilus influenzae type $\mathrm{b}$ conjugate vaccines in Alaska native infants. Pediatr Infect Dis J. 1993;12:484-492.

15. Brown VM, Madden S, Kelly L, Jamieson FB, Tsang RS, Ulanova M. Invasive Haemophilus influenzae disease caused by non-type b strains in Northwestern Ontario, Canada, 2002-2008. Clin Infect Dis. 2009;49:1240-3.

16. Kelly L, Tsang R, Morgan A, Jamieson F, Ulanova M. Invasive disease caused by Haemophilus influenzae type a in Northern Ontario First Nations communities. J Med Microbiol. 2011;60:384-390.

17. Nix EB, Williams K, Cox AD, St.-Michael F, Romero-Steiner S, Schmidt DS, McCready WG, Ulanova M. Naturally acquired antibodies against Haemophilus influenzae type a in Aboriginal adults, Canada. Emerg Infect Dis. 2015;21:273-9

18. Halperin SA, Scheifele D, Duval B, et al. Canadian Association for Immunization Research and Evaluation (CAIRE) Guidelines for industry sponsored clinical trial and epidemiology contract research. Hum Vaccines. 2005;140-2.

19. McNeil SA, Andrew MK, Ye L, Haguinet F, Hatchette TF, ElSherif M, LeBlanc J, Ambrose A, McGeer A, McElhaney JE, Loeb M, MacKinnon-Cameron D, Sharma R, Dos Santos G, Shinde V. Investigators of the Serious Outcomes Surveillance Network of the Canadian Immunization Research Network (CIRN). Interim estimates of 2014/15 influenza vaccine effectiveness in preventing laboratory-confirmed influenza-related hospitalisation from the Serious Outcomes Surveillance Network of the Canadian Immunization Research Network. Euro Surveill. 2015;20:21024.

20. Rubinstein E, Predy G, Sauvé L, Hammond GW, Aoki F, Sikora W, Li Y, Law B, Halperin S, Scheifele D. The responses of Aboriginal Canadians to adjuvanted pandemic (H1N1) 2009 influenza vaccine. CMAJ 2011;183:1033-1037. 